\title{
Deubiquitylase USP7 regulates human terminal erythroid differentiation by stabilizing GATA1
}

\author{
Long Liang, ${ }^{1,2}$ Yuanliang Peng, ${ }^{1}$ Jieying Zhang, ${ }^{1,3}$ Yibin Zhang, ${ }^{1,2}$ Mridul Roy, ${ }^{1,2}$ \\ Xu Han, ${ }^{1}$ Xiaojuan Xiao, ${ }^{1}$ Shuming Sun, ${ }^{1}$ Hong Liu, ${ }^{4}$ Ling Nie, ${ }^{4}$ Yijin Kuang, ${ }^{1}$ \\ Zesen Zhu, ${ }^{1}$ Jinghui Deng, ${ }^{1}$ Yang Xia, ${ }^{5}$ Vijay G. Sankaran, ${ }^{6,7}$ Christopher D. \\ Hillyer, ${ }^{8}$ Narla Mohandas, ${ }^{8}$ Mao Ye, ${ }^{2}$ Xiuli $\mathrm{An}^{3,9}$ and Jing Liu ${ }^{1,10}$
}

Haematologica 2019

Volume 104(11):2178-2188

\section{Correspondence:}

JING LIU

jingliucsu@hotmail.com or

liujing2@sklmg.edu.cn

XIULI AN

xan@nybc.org

MAO YE

goldleaf@hnu.edu.cn

Received: September 8, 2018.

Accepted: March 13, 2019.

Pre-published: March 14, 2019.

doi:10.3324/haematol.2018.206227

Check the online version for the most updated information on this article, online supplements, and information on authorship \& disclosures: www.haematologica.org/content/104/11/2178

\section{(C)2019 Ferrata Storti Foundation}

Material published in Haematologica is covered by copyright. All rights are reserved to the Ferrata Storti Foundation. Use of published material is allowed under the following terms and conditions:

https://creativecommons.org/licenses/by-nc/4.0/legalcode. Copies of published material are allowed for personal or internal use. Sharing published material for non-commercial purposes is subject to the following conditions:

https://creativecommons.org/licenses/by-nc/4.0/legalcode, sect. 3. Reproducing and sharing published material for commercial purposes is not allowed without permission in writing from the publisher.
${ }^{1}$ Molecular Biology Research Center \& Center for Medical Genetics, School of Life Sciences, Central South University, Changsha, China; ${ }^{2}$ Molecular Science and Biomedicine Laboratory, State Key Laboratory for Chemo/Biosensing and Chemometrics, College of Biology, College of Chemistry and Chemical Engineering, Hunan University, Changsha, China; ${ }^{3}$ Laboratory of Membrane Biology, New York Blood Center, New York, NY, USA; ${ }^{4}$ Xiangya Hospital, Central South University, Changsha, China; ${ }^{5}$ Department of Biochemistry and Molecular Biology, The University of Texas Health Science Center at Houston, Houston, TX, USA; ${ }^{6}$ Broad Institute of MIT and Harvard, Cambridge, MA, USA; 'Division of Hematology/Oncology, Boston Children's Hospital and Department of Pediatric Oncology, Dana-Farber Cancer Institute, Harvard Medical School, Boston, MA, USA; ${ }^{8}$ Red Cell Physiology Laboratory, New York Blood Center, New York, NY, USA; ' 5 chool of Life Sciences, Zhengzhou University, Zhengzhou, China and ${ }^{10}$ Erythropoiesis Research Center, Central South University, Changsha, China

\section{ABSTRACT}

U biquitination is an enzymatic post-translational modification that affects protein fate. The ubiquitin-proteasome system (UPS) was first discovered in reticulocytes where it plays important roles in reticulocyte maturation. Recent studies have revealed that ubiquitination is a dynamic and reversible process and that deubiquitylases are capable of removing ubiquitin from their protein substrates. Given the fact that the UPS is highly active in reticulocytes, it is speculated that deubiquitylases may play important roles in erythropoiesis. Yet, the role of deubiquitylases in erythropoiesis remains largely unexplored. In the present study, we found that the expression of deubiquitylase USP7 is significantly increased during human terminal erythroid differentiation. We further showed that interfering with USP7 function, either by short hairpin RNA-mediated knockdown or USP7-specific inhibitors, impaired human terminal erythroid differentiation due to decreased GATA1 level and that restoration of GATA1 levels rescued the differentiation defect. Mechanistically, USP7 deficiency led to a decreased GATA1 protein level that could be reversed by proteasome inhibitors. Furthermore, USP7 interacts directly with GATA1 and catalyzes the removal of K48-linked polyubiquitylation chains conjugated onto GATA1, thereby stabilizing GATA1 protein. Collectively, our findings have identified an important role of a deubiquitylase in human terminal erythroid differentiation by stabilizing GATA1, the master regulator of erythropoiesis.

\section{Introduction}

Red blood cells, the most abundant of all circulating blood cells, facilitate gas exchange in the lungs and transporting oxygen to tissues. More than two million red blood cells are generated per second in a healthy adult through a process termed erythropoiesis. Mature red blood cells are produced from hematopoietic stem cells, which commit to erythroid progenitors followed by terminal erythroid differentiation. Terminal erythroid differentiation, driven by the glycoprotein hormone erythropoietin, begins with proerythroblasts, which sequentially divide into basophilic, polychromatic and orthochromatic erythroblasts that enucleate to generate reticulocytes. ${ }^{1,2}$ Erythropoiesis is a tightly regulated process. Previous studies were primarily focused on the regulation of erythropoiesis by transcription factors and cytokines. ${ }^{3,4}$ 
In contrast, the regulation of erythropoiesis by other mechanisms has been less well studied. Notably, our knowledge on post-translational regulation of erythropoiesis is limited.

Ubiquitination is an enzymatic post-translational modification. Ubiquitinated proteins are degraded by the ubiquitin-proteasome system (UPS). The UPS controls the degradation of most intracellular proteins and plays important roles in many cellular processes..$^{5}$ Although the UPS was first discovered in reticulocytes over 40 years ago, ${ }^{6}$ to date there are only limited studies on the roles of the UPS in erythropoiesis. These include the reported role of CUL4A-mediated degradation of p27 in cell proliferation in the early stages of erythropoiesis and cell cycle exit at a later stage of erythropoiesis. ${ }^{7,8}$ A recent, exciting study demonstrated that UBE2O remodels the proteome during terminal erythroid differentiation, underscoring the importance of the UPS in erythropoiesis. ${ }^{9}$

Ubiquitination is a dynamic and reversible process. ${ }^{10}$ It has been reported that deubiquitylases are capable of removing ubiquitin from their protein substrates and allow proteins to be salvaged from proteasomal degradation. ${ }^{11}$ USP7 is a deubiquitylase that belongs to the ubiquitin-specific protease (USP) family, which constitutes the largest subgroup of deubiquitylases. Accumulated evidence has shown that USP7 plays diverse roles in genome stability, epigenetic regulation, the cell cycle, apoptosis, viral infection, immunity and stem cell maintenance. ${ }^{12-17}$ Recently, USP7 was reported to be an important regulator of osteogenic differentiation and adipogenesis. ${ }^{18,19}$ Our RNA-sequencing analyses revealed highlevel expression of genes/pathways (including USP7) involved in the ubiquitin system during late stages of terminal erythroid differentiation. ${ }^{2}$ Nevertheless, the function of USP7 in human erythropoiesis remains unexplored.

GATA1 is the key transcription factor for erythropoiesis, controlling the expression of a large series of erythroid genes, including erythropoietin receptor, globins and several membrane proteins. ${ }^{20}$ GATA1-deficient mice die in utero due to severe anemia at embryonic day $10.5-11.5,{ }^{21}$ and chimeric mice lacking GATA1 fail to produce mature red blood cells, although the formation of cells of other hematopoietic lineages is normal. ${ }^{22}$ In contrast, overexpression of GATA1 in erythroid cells inhibits their differentiation, leading to fatal anemia in mice. ${ }^{23}$ GATA1 stability is finely regulated by multiple mechanisms ${ }^{24}$ since changes in its protein levels will exert a great influence on erythropoiesis. Although GATA1 degradation by the ubiquitin-proteasome pathway has been characterized, ${ }^{24}$ how GATA1 recycles from the UPS is yet to be defined.

In this study, we demonstrated that USP7 deficiency impairs human terminal erythroid differentiation due to a decreased level of GATA1 protein. We further showed that USP7 interacts directly with GATA1 and catalyzes the removal of poly-ubiquitylation chains on GATA1, thus stabilizing GATA1. Our findings have thus not only documented the role of a deubiquitylase in erythropoiesis, but also enabled the identification of a novel mechanism by which deubiquitylases regulate GATA1 protein stability.

\section{Methods}

\section{Reagents and antibodies}

P5091 (S7132) and MG132 (S2619) were obtained from Selleckchem (TX, USA); P22077 (HY-13865) from MCE (NJ,
USA); and cycloheximide was purchased from Sigma-Aldrich (MO, USA). Antibodies used for western blot, immunoprecipitation and immunofluorescence studies are detailed in the Online Supplementary Methods. The antibodies used for flow cytometry analysis were glycoprotein A (GPA)-PE-Cy7, GPA-APC, and $\alpha 4$ integrin (CD49d)-PE from BD Pharmingen (NJ, USA). Band 3$\mathrm{APC}$ and $4.1 \mathrm{R}$ antibodies were used as previously described. ${ }^{25}$

\section{Cell culture}

Human cord blood samples were obtained from Xiangya Hospital of Central South University or New York Blood Center under Institutional Review Board approval and in accordance with the Declaration of Helsinki. The detailed composition of the culture medium and the cell culture protocol has been described previously. ${ }^{25}$ HEK293T cells (American Type Culture Collection: CRL-11268) were cultured in Dulbecco modified Eagle medium (Gibco, MA, USA) supplemented with 10\% fetal bovine serum (Gibco).

\section{Lentivirus packaging and infection}

USP7-specific short hairpin (sh)RNA was purchased from GenePharma (Shanghai, China) (shRNA \#1: 5'AGTCGTTCAGTCGTCGTAT-3' and \#2: 5'-TGGATTTGTGGTTACGTTACTC-3', constructed in pGLV3-H1-GFP or pGLV2U6 vector). GATA1 overexpression (HMD-GATA1-IRES-GFP) and control plasmids have been described previously. ${ }^{26}$ Lentiviruses were packaged in HEK293T cells according to the

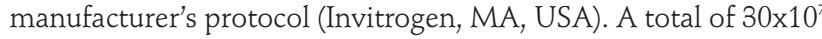
lentiviral particles were infected using polybrane with $0.5 \times 10^{7}$ CD34 $4^{+}$cells on day 3 or 4 . Puromycin $(1 \mu \mathrm{g} / \mathrm{mL})$ was used for selection of transduced cells.

\section{GATA1 rescue assay}

For rescue experiments, erythroid cells were infected with USP7 shRNA or control shRNA lentiviruses for 3 days. On day 7 of culture, erythroid cells were transduced with the control or GATA1 lentivirus. Double-transduced cells were identified following puromycin $(1 \mu \mathrm{g} / \mathrm{mL})$ selection and GFP expression from the HMD vector. The extent of terminal erythroid differentiation was monitored beginning on day 9 .

\section{RNA isolation, quantitative real-time polymerase chain reaction and western blot analysis.}

Standard protocols were used for RNA and protein isolation, polymerase chain reaction (PCR) and western blot analysis. Details are given in the Online Supplementary Methods. The GATA1 primer sequences were described previously. ${ }^{27}$ USP7 primer sequences were: forward: 5'-AGCGTGGCATCACCATAATC-3' and reverse: 5'-CGAGGCAACCTTTCAGTTCA-3'.

\section{Immunoprecipitation and glutathione-S-transferase pull-down}

Immunoprecipitation studies were performed using M2/Flag or protein A/G-agarose beads. For the glutathione-S-transferase (GST) pull-down assay, purified Flag-USP7 and bacterial expressed GST or GST-GATA1 were used. The methods are described in detail in the Online Supplementary Methods.

\section{In vivo ubiquitylation and deubiquitylation assays}

For cell-based deubiquitylation assays, Flag-GATA1 and HAubiquitin were co-transfected with an empty vector or a vector expressing USP7 (WT or CS) for $48 \mathrm{~h}$. For USP7 knockdown, the cells were infected with the lentiviruses for $48 \mathrm{~h}$. Additional details of the methods are given in the Online Supplementary Methods. 


\section{In vitro deubiquitylation assays}

In vitro ubiquitylation assays were performed as previously described $^{28}$ and additional details are provided in the Online Supplement.

\section{Statistical analysis}

All data are presented as mean \pm standard deviation (SD), and the results were analyzed using the SPSS 18.0 software package. Significant differences between groups were determined using analysis of variance and the Tukey range test.

\section{Results}

\section{Deficiency of USP7 impairs human terminal erythroid differentiation}

To explore the roles of deubiquitylases during erythropoiesis, we first analyzed the expression patterns of deubiquitylases in human erythroblasts at different stages of differentiation from our RNA-sequencing data. ${ }^{2}$ Figure 1A shows the expression patterns of USP family members and reveals that the expression levels of USP7 are significantly increased during erythropoiesis. Based on the previously identified important role of USP7 in cell differentiation in other cellular systems, ${ }^{17-19}$ in the present study we focused our attention on the role of USP7 in erythroid differentiation. We confirmed the increased expression of USP7 during late stages of erythroid differentiation by both real-time PCR (Figure 1B) and by western blot analysis (Figure 1C). To examine the effect of USP7 on erythropoiesis, we employed a shRNA-mediated knockdown approach in human CD34+ ${ }^{+}$cells. ${ }^{27,29,30}$ As shown in Figure 1D, USP7 knockdown impaired the terminal erythroid differentiation as demonstrated by the decreased surface expression of the erythroid marker GPA, delayed loss of a4-integrin expression in association with decreased surface expression of band 3. There was also a marked decreased in the extent of enucleation. USP7 knockdown also inhibited the expression of hemoglobin (Figure 1E). The significant impairment of the growth of late-stage erythroblasts caused by USP7 knockdown was accompanied by increased apoptosis (Online Supplementary Figure S1A, B). Similar to USP7 knockdown, USP7-specific inhibitors P5091 and P22077 ${ }^{31,32}$ also impaired human terminal erythroid differentiation, inhibited hemoglobin expression (Figure 1F, G) and cell proliferation (Online Supplementary Figure $S 1 C, D$ ). These results imply that USP7 plays an important role in human terminal erythroid differentiation.

\section{USP7 regulates erythroid differentiation by modulating GATA1 protein levels}

We subsequently explored the molecular mechanism(s) of the altered erythropoiesis due to USP7 deficiency. Given the fact that USP7 functions in the nucleus, ${ }^{33,34}$ we hypothesized that USP7 might affect erythropoiesis by regulating erythroid differentiation-related transcription factors. As shown in Figure 2A and Online Supplementary Figure S2, GATA1 was the transcription factor most significantly decreased after knockdown of USP7, although KLF1 levels also decreased. Since KLF1 expression is regulated by GATA $1{ }^{35}$ we suggest that the decreased expression of KLF1 is a consequence of GATA1 downregulation. Interestingly, mRNA levels of GATA1 were not affected by USP7 knockdown on day 9 (Figure 2B), suggesting that the observed decrease in GATA1 levels is at the post-transcriptional level. We noted decreased levels of GATA1 mRNA levels on days 11 and 13 following USP7 knockdown (Online Supplementary Figure S3A, B), likely due to the fact that GATA1 mediates its own regulation at the late stages of erythropoiesis. ${ }^{36,37}$ Similarly, inhibition of USP7 activity by the USP7-specific inhibitors P5091 and P22077 also resulted in significant decreases in GATA1 protein levels in a dose-dependent manner (Figure 2C, D), with no obvious effects on other transcription factors beside a slightly decreased expression of KLF1 (Online Supplementary Figure S4), implying that USP7-mediated regulation of GATA1 depends on the enzymatic activity of USP7. To further confirm that USP7 deficiency-induced defective erythropoiesis is due to downregulation of GATA1, we performed rescue experiments by ectopically expressing GATA1 in USP7 knockdown cells. Figure 2E shows that the delayed erythroid differentiation as well as impaired erythroblast enucleation could be rescued by restoring GATA1 levels. Furthermore, the expression of GATA1 target genes such as 4.1R and HBG were also rescued (Figure 2F). These results imply that USP7 regulates erythroid differentiation through GATA1.

\section{USP7 regulates the stability of the GATA1 protein}

GATA1 protein levels are regulated by several proteins, including HSP70, a GATA1 chaperone ${ }^{38-40}$ and RPS19, necessary for GATA1 translation. ${ }^{26,41}$ To examine whether HSP70 and RPS19 are involved in the regulation of GATA1 mediated by USP7, we analyzed the effect of USP7 knockdown on their expression levels. As shown in Online Supplementary Figure S5A, B, USP7 knockdown or inhibition had no effects on HSP70 or RPS19 protein levels. Moreover, USP7 knockdown did not affect the translocation of HSP70 into the nucleus (Online Supplementary Figure S5C, D). The above findings strongly suggest that USP7 regulates the stability of GATA1 protein directly. We performed several additional studies to confirm this hypothesis and to define the underlying mechanisms. First, coexpression of wildtype USP7 (USP7-WT) with GATA1 increased the GATA1 level (Figure 3A). Importantly, catalytically inactive mutant USP7 (USP7-CS, C233S) did not increase GATA1 protein levels (Figure 3B). Second, downregulation of GATA1 by USP7 knockdown or the USP7 inhibitors P5091 and P22077 was reversed by the proteasome inhibitor MG132 (Figure 3C-E), implying that USP7 maintains the steady-state levels of GATA1 by blocking its proteasomal degradation. To further examine the relationship between USP7 and GATA1, we measured the half-life of intracellular GATA1 after cells had been treated with cycloheximide to inhibit protein biosynthesis. As shown in Figure 3F, knockdown of USP7 significantly shortened the half-life of the GATA1 protein. Conversely, overexpression of USP7-WT, but not USP7-CS, prolonged the half-life of GATA1 (Figure 3G). Taken together, our data demonstrate that USP7 stabilizes GATA1 by preventing its proteasomal degradation.

\section{USP7 interacts directly with GATA1}

Having demonstrated that USP7 stabilizes GATA1, we then examined whether this effect is through their direct interaction by performing co-immunoprecipitation experiments. USP7 or GATA1 was separately immunoprecipitated from cultured primary erythroblasts and the reciprocal protein was detected by western blot analysis. As 
A

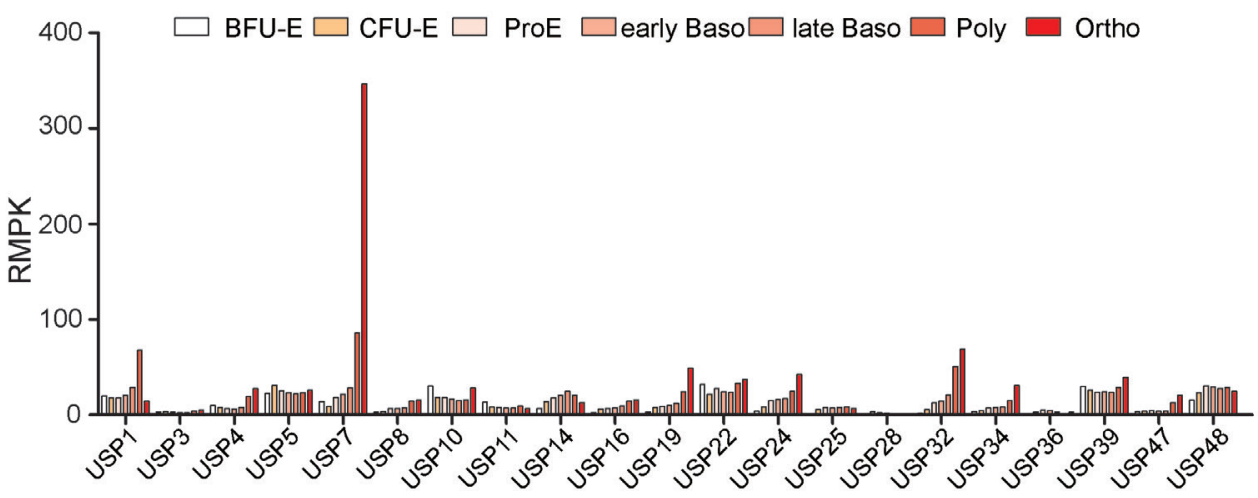

B

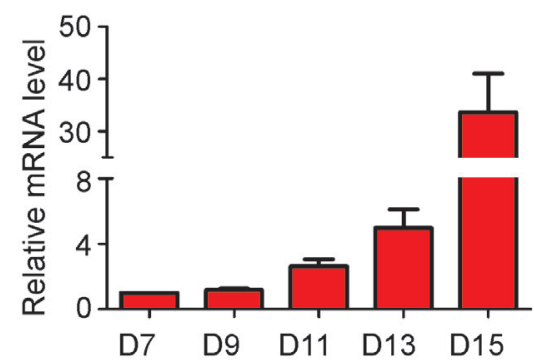

D

D9

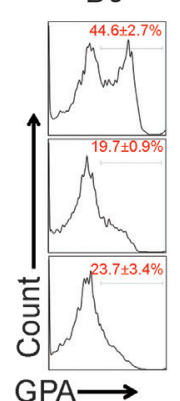

$\mathbf{F}$

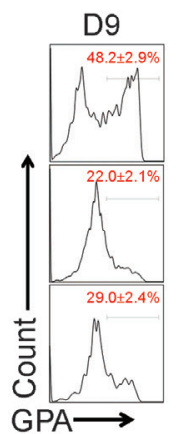

D11

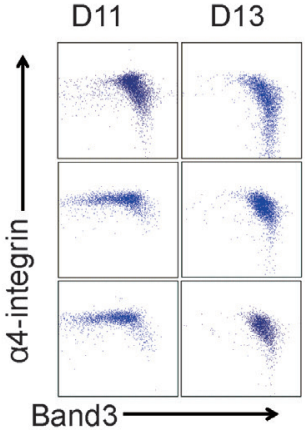

D11 D13

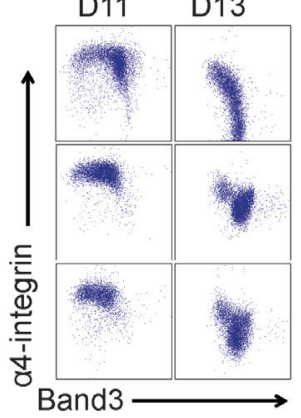

C

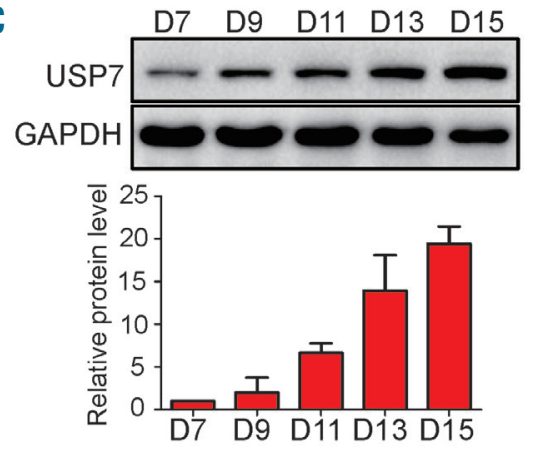

D13
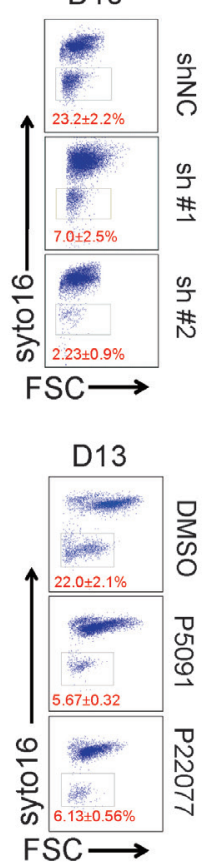

E

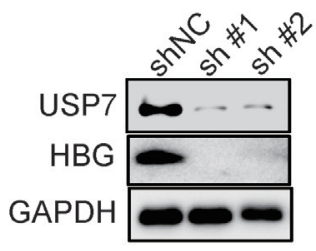

G

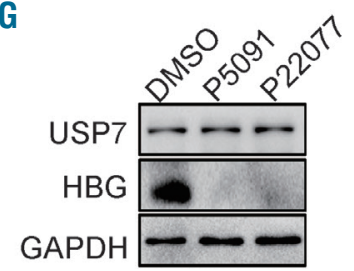

Figure 1. Deficiency of USP7 impairs human terminal erythroid differentiation. (A) RNA-sequencing data showing the expression of USP family members (fragments per kilobase of transcript per million) at each distinct stage of human terminal erythroid differentiation. (B) Real-time quantitative polymerase chain reaction results showing the expression of USP7 mRNA on the indicated days of human erythroid terminal differentiation. (C) Representative western blot analysis of the protein level of USP7 on the indicated days of human terminal erythroid differentiation. Quantitative analysis of data from three independent experiments of protein expression levels are shown (lower panel). (D) Left, representative profiles of flow cytometry-based detection of glycophorin A (GPA) expression in erythroblasts infected with the control or USP7 shRNA on day 9 . Middle, representative profiles of Band3/ $\alpha 4$-integrin levels of GPA-positive erythroblasts transfected with the control or USP7 shRNA lentviruses on days 11 and 13. Right, representative profiles of flow cytometry-based detection of enucleation by syto16 staining on day 13 . Quantification from three independent experiments is indicated. (E) Representative western blot showing the protein level of $\gamma$-hemoglobin (HBG) and USP7 in erythroblasts transfected with either the control or USP7 shRNA on day 9. (F) Left, representative profiles of flow cytometry-based detection of GPA expression in erythroblasts treated with dimethylsulfoxide (DMSO) or USP7 inhibitor P5091 (5 uM) or P22077 (7.5 $\mu \mathrm{M})$ on day 9. Middle, representative profiles of Band3/ $\alpha 4$-integrin levels of GPA-positive erythroblasts treated with DMSO or USP7 inhibitor P5091 or P22077 on day 11 and day 13. Right, representative profiles of flow cytometry-based detection of enucleation by syto16 staining on day 13. Quantification from three independent experiments is shown. (G) Representative western blot showing the protein level of HBG and USP7 in erythroblasts treated with DMSO or USP7 inhibitors (P5091 or P22077) on day 9. For all western blot analyses, GAPDH was used as the loading control. BFU-E: burst-forming unit - erythroid; CFU-E: colony-forming unit - erythroid; ProE: proerythroblast; early Baso: early basophilic erythroblast; late Baso: late basophilic erythroblast; Poly: polychromatic erythroblast; Ortho: orthochromatic erythroblast; D: day; GPA: glycophorin A; FSC: forward scatter; DMSO: dimethylsulfoxide. 
A

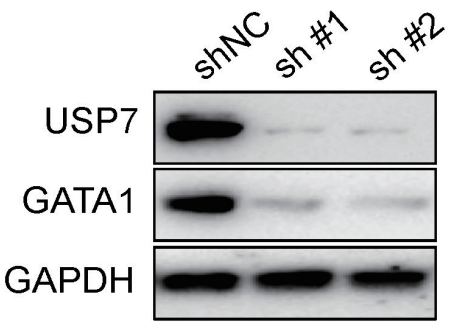

C
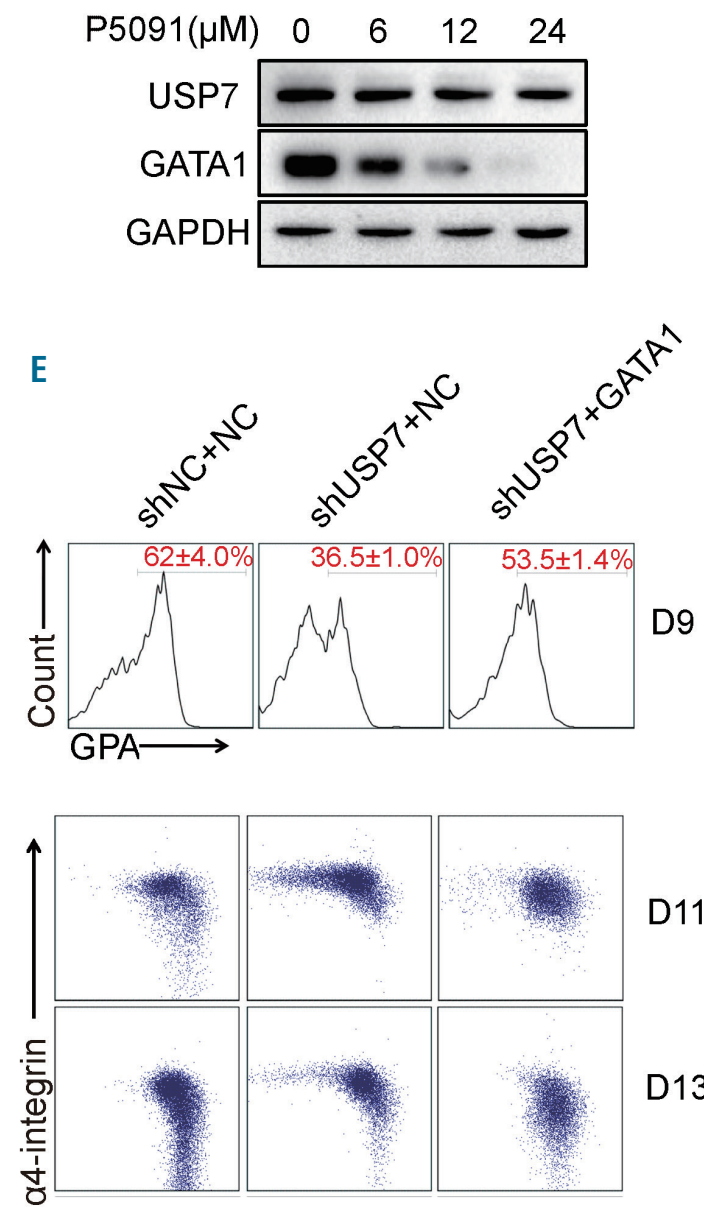

D11

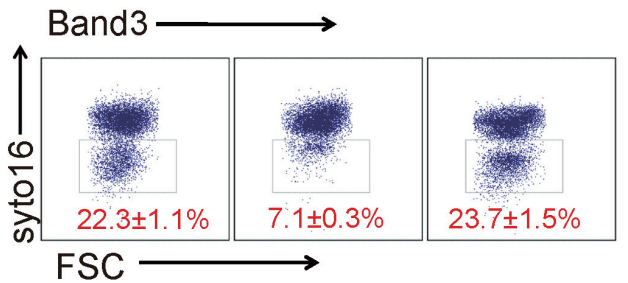

B

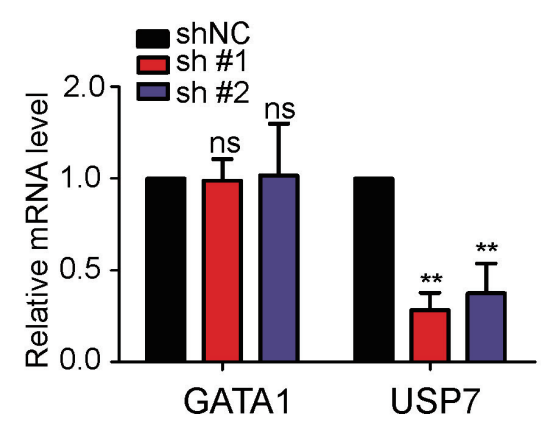

$\mathrm{P} 22077(\mu \mathrm{M}) \quad 0 \quad 6 \quad 12 \quad 24$

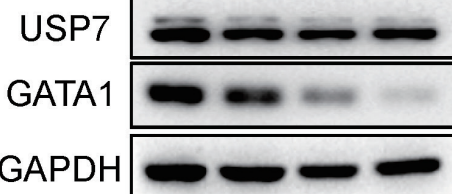

$\mathbf{F}$

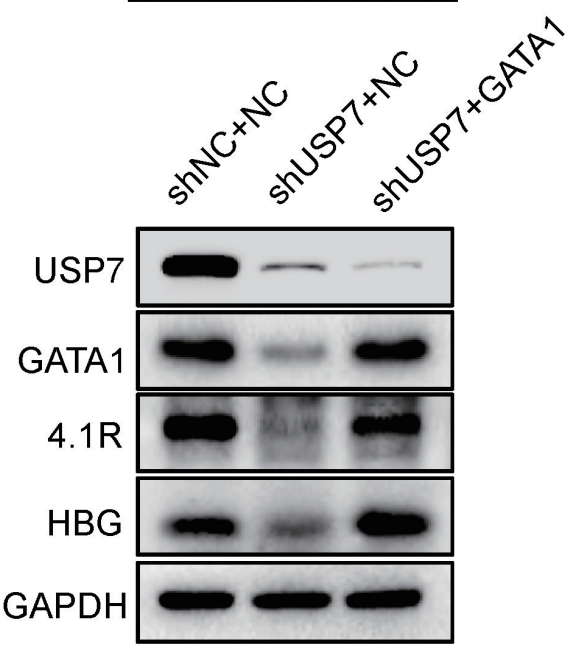

Figure 2. USP7 regulates erythroid differentiation by affecting GATA1 protein levels. (A) Representative western blot analysis of erythroblasts transfected with control or USP7 short hairpin (sh)RNA on day 9. GAPDH was used as a loading control. (B) Bar graph presentation of USP7 and GATA1 mRNA levels as determined by real-time quantitative polymerase chain reaction analysis of erythroblasts transfected with the negative control shRNA or USP7 shRNA \#1 or \#2, which were harvested on day 9. (C) Representative western blot analysis of the erythroblasts after treatment with indicated doses of P5091 on day 9. GAPDH was used as the loading control. (D) Representative western blot analysis of the erythroblasts after treatment with different doses of P22077 on day 9. GAPDH was used as the loading control. (E) The upper panel shows the representative profiles of flow cytometry analysis of GPA expression on day 9 in erythroblasts transfected with control shRNA and NC (HMD empty vector), USP7 shRNA and NC, or USP7 shRNA and GATA1 (GATA1-HMD). The middle panel shows the representative profiles of flow cytometry analysis of Band3/ $\alpha 4$-integrin expression of the GPA-positive cells in the same groups on days 11 and 13 . The bottom panel shows the representative profiles of flow cytometry-based detection of enucleation by syto16 staining on day 13. Quantification from three independent experiments is indicated. (F) Representative western blot analysis of erythroblasts transfected with control shRNA and NC (HMD empty vector), USP7 shRNA and NC, or USP7 shRNA and GATA1 (GATA1-HMD) on day 9. (G) Bar diagram presenting the quantitative analysis of protein expression data from (F). The plot was generated from three independent experiments and shows the means \pm standard deviations $(* * P<0.01, * P<0.05)$. 
shown in Figure 4A, endogenous USP7 was immunoprecipitated by anti-GATA1 antibodies but not by control IgG. Conversely, GATA1 was immunoprecipitated by anti-USP7 antibodies but not by control IgG (Figure 4B). To determine whether USP7 and GATA1 interact directly with each other, we performed GST pull-down assays under a cell-free condition by using purified recombinant GST-GATA1 and Flag-USP7 proteins. As shown in Figure 4C, the purified GST-GATA1 but not the control GST was able to pull down USP7. Furthermore, we mapped the detailed binding region of GATA1 and USP7 in
HEK293T cells, a non-erythroblast environment. ${ }^{38,42,43}$ Truncation mutants of GFP-USP7 and Flag-GATA1 were co-transfected into HEK293T cells and co-immunoprecipitation analyses revealed that the $\mathrm{N}$-terminal TRAF-like domain (1-208) of USP7 was critical for the interaction between GATA1 and USP7 (Figure 4D). Conversely, mapping the region of GATA1 required for USP7 binding showed that the DNA binding domain (200-290) of GATA1 was responsible for its interaction with USP7 (Figure 4E). Collectively, these results show that USP7 interacts with GATA1 directly.

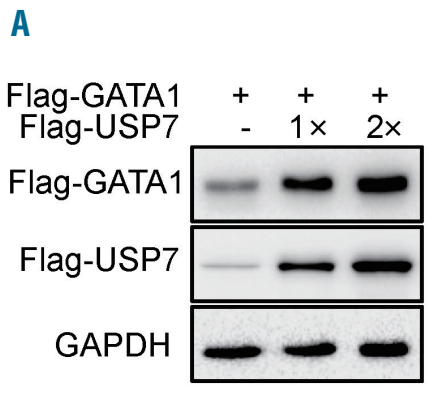

D

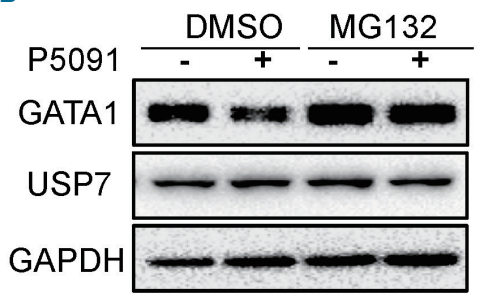

B
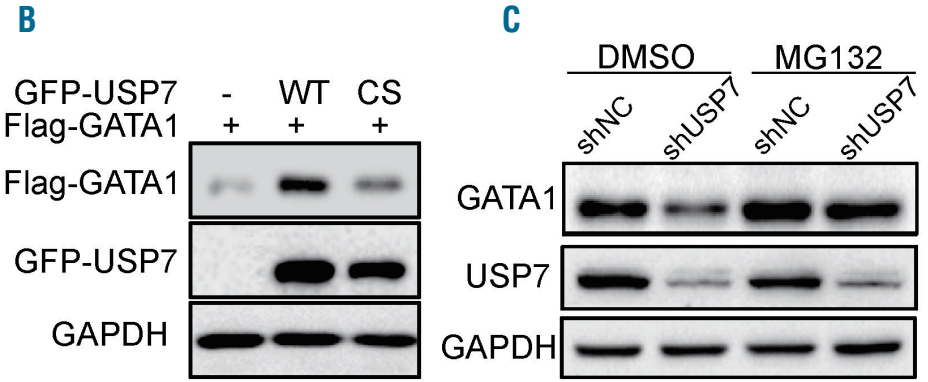

$E$

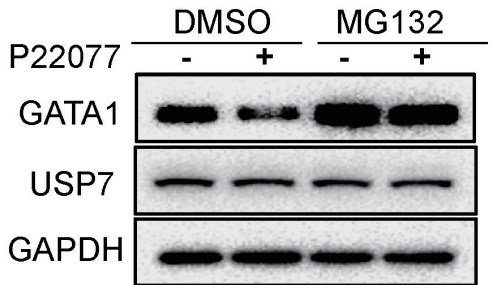

$\mathbf{F}$

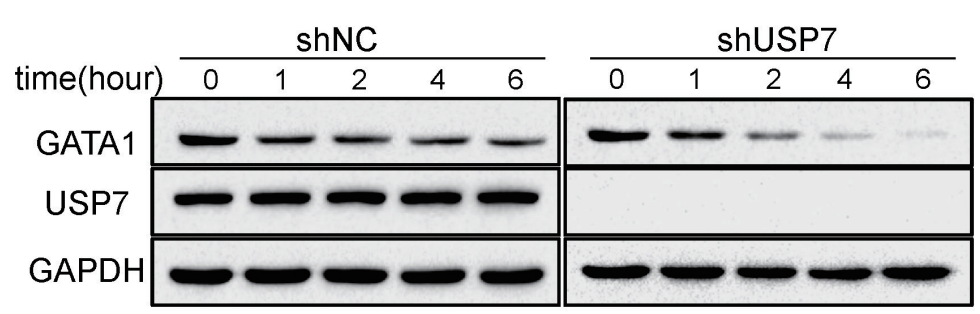

G

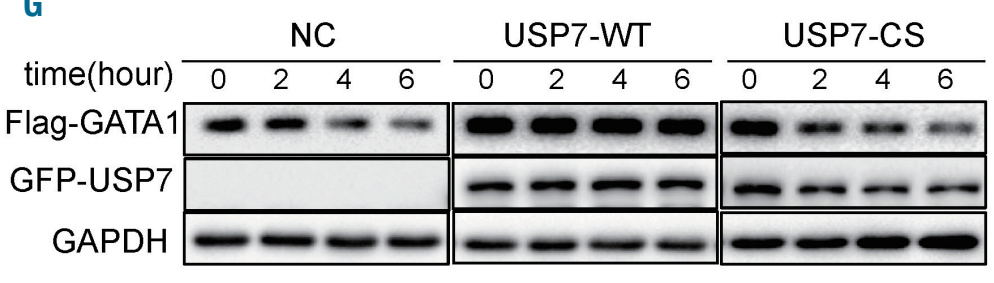

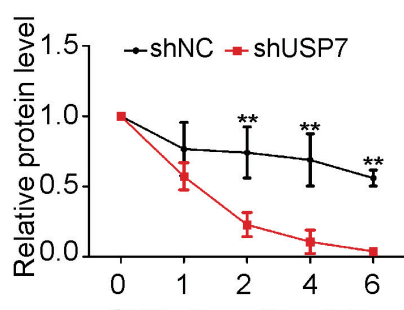

$\mathrm{CHX}$-chase time $(\mathrm{h})$

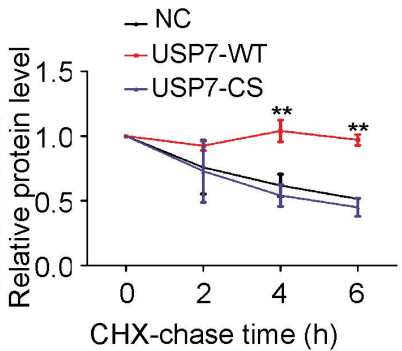

Figure 3. USP7 regulates the stability of GATA1. (A) Representative western blot analysis of HEK293T cells transfected with a GATA1-expressing plasmid and either an empty vector or increasing amounts of a GFP-USP7-expressing vector. (B) Representative western blot analysis of HEK293T cells that were transfected with a construct expressing Flag-GATA1 and an empty vector (-), a construct expressing wildtype USP7 (USP7-WT) or one expressing the USP7 (Cys223Ser) mutant (USP7CS). (C) Representative western blot analysis of erythroblasts that were transfected with control (shNC) or USP7 shRNA treated with or without the proteasome inhibitor MG132 (20 $\mu \mathrm{M}, 6 \mathrm{~h}$ ). (D) Representative western blot analysis of erythroblasts that were treated for $6 \mathrm{~h}$ with dimethylsulfoxide (DMSO) (-) and/or $15 \mu \mathrm{M}$ P5091 (+) or with 15 uM P5091 and 20 uM MG132. (E) Representative western blot for the expression of GATA1 in erythroblasts treated for $6 \mathrm{~h}$ with DMSO (-) and/or $15 \mu \mathrm{M}$ P22077 (+) or with $15 \mu \mathrm{M}$ P22077 and $20 \mu \mathrm{M}$ MG132 on day 9. (F) Erythroblasts transfected with control or USP7 shRNA were treated with cycloheximide $(\mathrm{CHX})(150 \mu \mathrm{g} / \mathrm{mL})$, and collected at the indicated times for western blot. Results are shown as mean \pm standard deviation (SD) (**P<0.01). (G) Representative western blot analysis of HEK293T cells that were transfected with a vector expressing Flag-GATA1 and an empty vector (NC), one expressing GFP-USP7-WT or one expressing GFP-USP7-CS, after treatment with $\mathrm{CHX}(150 \mu \mathrm{g} / \mathrm{mL})$ for the indicated amounts of time. Results are shown as mean \pm standard deviation $(* \star P<0.01)$. For all western blot analyses, GAPDH was used as the loading control. 
USP7 stabilizes GATA1 protein through K48 deubiquitylation

Since USP7 is a deubiquitylase, it is reasonable to speculate that USP7 regulates the stability of GATA1 via deubiquitylation. To test this hypothesis, we investigated the effect of USP7 on the poly-ubiquitylation of GATA1. As expected, knockdown of USP7 resulted in a significant increase in the poly-ubiquitylation of GATA1 (Figure 5A). In contrast, ectopic expression of USP7-WT but not the catalytic inactive mutant USP7-CS reduced the level of poly-ubiquitylation of GATA1 (Figure $5 \mathrm{~B}$ and Online Supplementary Figure S6A). Moreover, USP7-mediated decrease of GATA1 poly-ubiquitylation was blocked by the USP7 inhibitors P5091 and P22077 (Figure 5C and Online Supplementary Figure S6B), demonstrating that the enzymatic activity of USP7 is essential for the USP7-mediated deubiquitylation of GATA1. To verify that GATA1 is a direct substrate of USP7, the purified USP7 protein was incubated with ubiquitylated GATA1 in a cell-free system. As shown in Figure 5D, GATA1 poly-ubiquitylation was decreased in the presence USP7, indicating that USP7 deubiquitylates GATA1 directly. To determine which polyubiquitin chain on GATA1 is removed by USP7, we employed a series of ubiquitin mutants that contain only
A

\begin{tabular}{llll}
\cline { 2 - 3 } & $\frac{\text { Input }}{\text { D9 D11 D13 }} \frac{\text { IgG }}{\text { D9 D11 D13 }} \frac{\text { IP:GATA1 }}{\text { D9 D11 D13 }}$ \\
USP7 & $-\infty$ & \\
GATA1 & $-\infty$ &
\end{tabular}

B $\frac{\text { Input }}{\text { D9 D11 D13 }} \frac{\text { IgG }}{\text { D9 D11 D13 D9 D11 D13 }}$

\begin{tabular}{l|ll} 
GATA1 & & \\
USP7 & $-\infty-\infty$ &
\end{tabular}

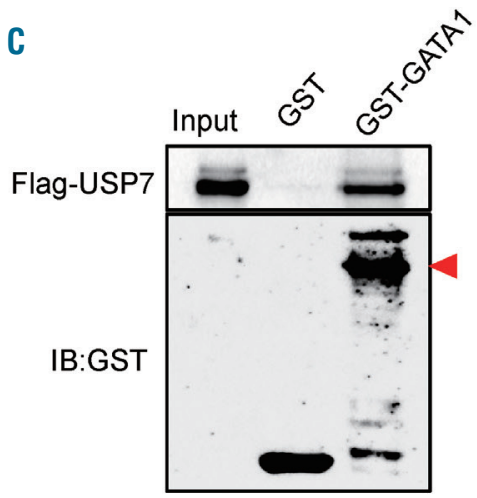

Figure 4. USP7 interacts directly with GATA1. (A) Representative western blot analysis of USP7 after immunoprecipitation of endogenous GATA1 at the indicated time-points (days 9, 11 and 13) from erythroblasts. (B) Representative western blot analysis of GATA1 after immunoprecipitation of endogenous USP7 at the indicated time-points (days 9, 11 and 13) from erythroblasts. (C) Representative western blot analysis of pull-down of purified Flag-USP7 with purified GST-GATA1. (D) Schematic representation of the USP7 deletion mutants used for domain mapping (left panel). HEK293T cells ectopically expressing GATA1 were co-transfected with USP7 deletion mutants. Interactions were analyzed using a co-immunoprecipitation assay (right panel). (E) Schematic representation of full length and various deletion mutants of GATA1 used for domain mapping (left panel). HEK293T cells ectopically expressing USP7 were co-transfected with GATA1 deletion mutants. Interactions were analyzed using a co-immunoprecipitation assay (right panel)
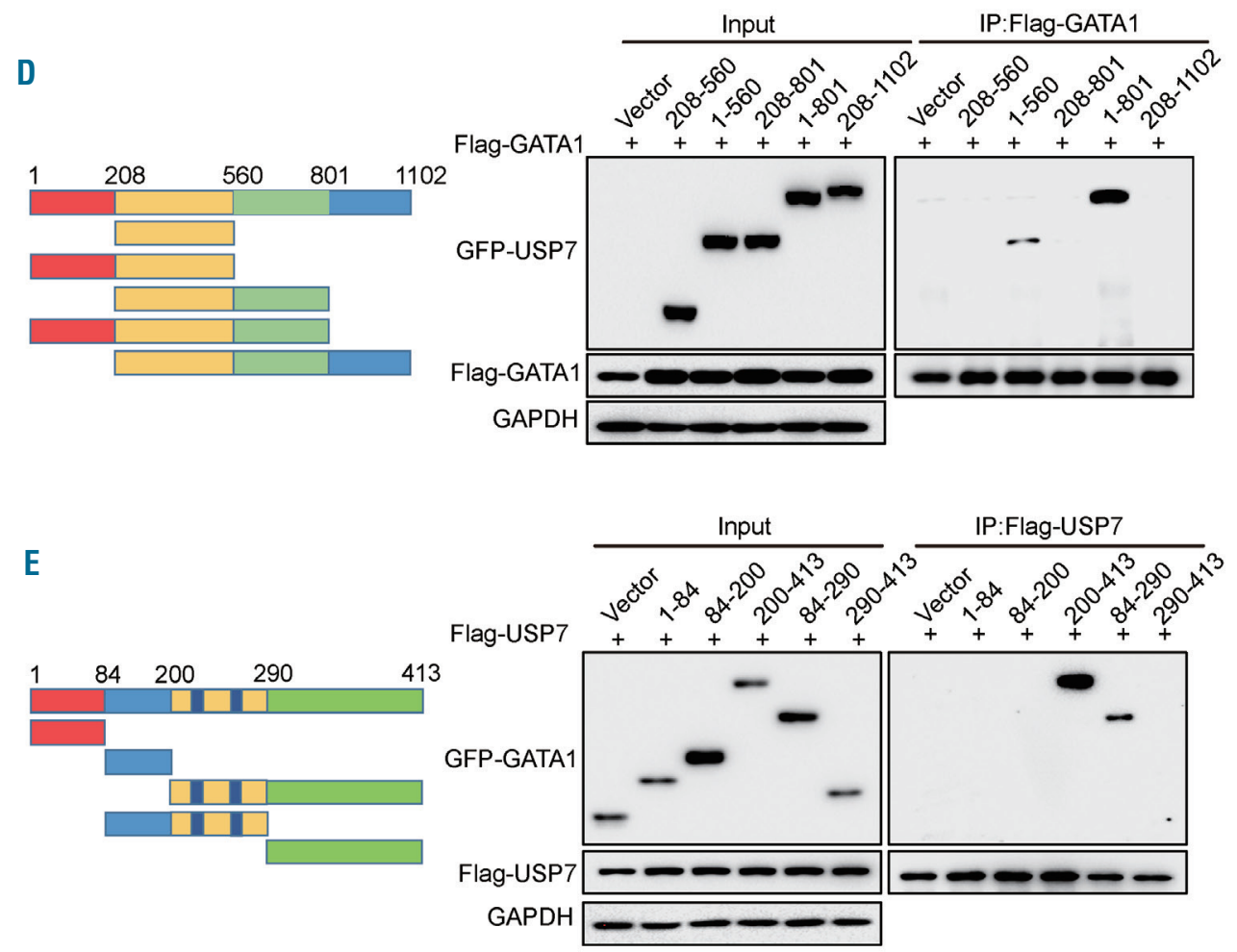
one lysine (K6, K11, K27, K29, K33, K48 or K63). As shown in Online Supplementary Figure S7, USP7 significantly decreased only the K48-linked poly-ubiquitin chain but not any other lysine isopeptide-linked poly-ubiquitin chains (K6, K11, K27, K29, K33 or K63). To further confirm that K48-linked poly-ubiquitin is removed by USP7, we replaced K48 or K63 lysine by arginine (R) and, as shown in Figure 5E, mutation of K48 but not K63 significantly impaired USP7-mediated deubiquitylation. Collectively, these results confirm that USP7 stabilizes GATA1 by removing the K48-linked poly-ubiquitin.

\section{Discussion}

Erythropoiesis is a process by which hematopoietic stem cells proliferate and differentiate to eventually produce mature red blood cells. Many cellular and molecular changes occur during this process. Morphological changes include a progressive decrease in cell size, increase in chromatin condensation and enucleation. At the molecular level, high-throughput analyses revealed dramatic changes in both the transcriptome and the proteome. ${ }^{2,44}$ In contrast to extensive studies on transcriptional networks, very little
A

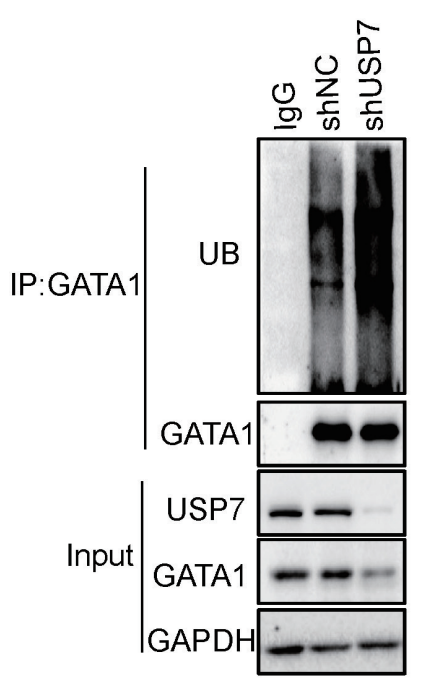

B

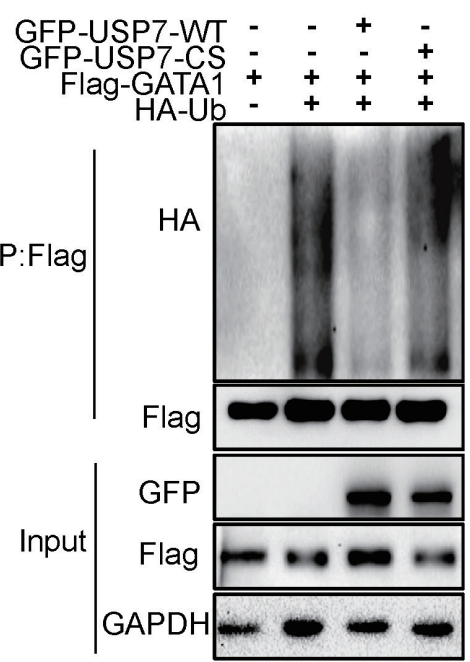

C

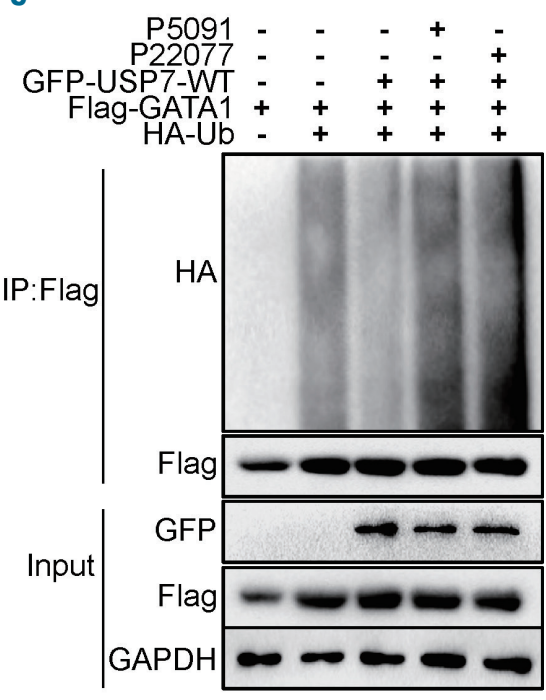

D

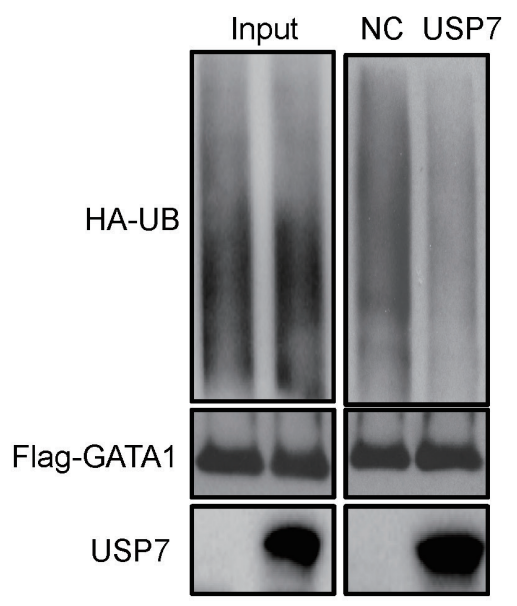

E

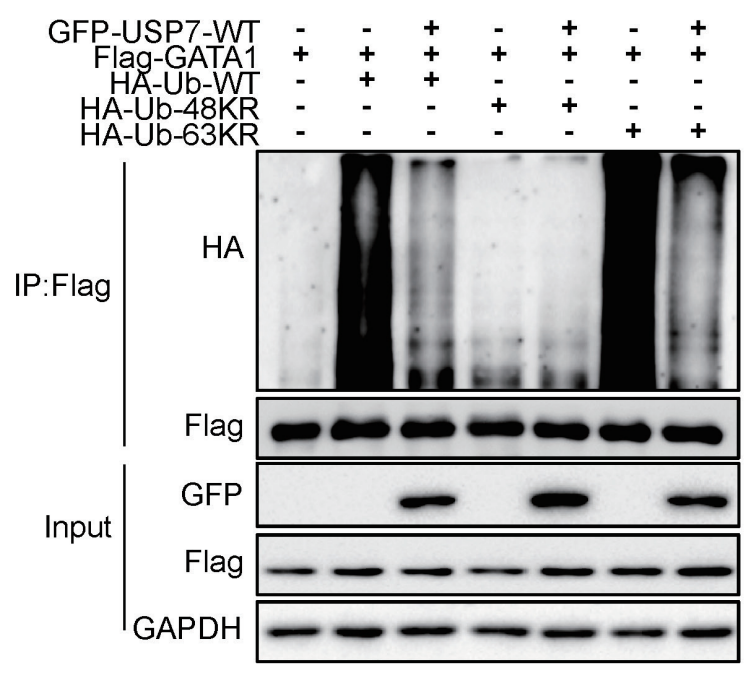

Figure 5. USP7 stabilizes GATA1 protein through deubiquitination. (A) Erythroblasts at day 7 transfected with control or USP7 shRNA (\#1) lentivirus. GATA1 was immunoprecipitated with anti-GATA1 anyibody and immunoblotted with anti-ubiquitin on day 9. (B) Representative western blot analysis of ubiquitin after incubation of anti-Flag-coated beads with lysates from HEK293T cells that were transfected with empty vectors (-) or those expressing Flag-GATA1 either alone or in combination with vectors expressing USP7-WT or USP7-CS, and HA-ubiquitin. (C) Representative western blot analysis for ubiquitin after anti-Flag immunoprecipitation of HEK293T cells ectopically expressing Flag-GATA1 either alone or in combination with USP7-WT. Cells expressing both Flag-GATA1 and USP7-WT were treated with $20 \mu \mathrm{M}$ P5091 or P22077 for $8 \mathrm{~h}$ before being harvested. (D) Representative western blot for the cell-free deubiquitylation assay. Ubiquitylated GATA1 was incubated with bacterial-expressed and purified USP7-WT for $2 \mathrm{~h}$ at $37^{\circ} \mathrm{C}$, followed by western blot with anti-HA antibody (right panel). The left panel is the input. (E) Representative western blot analysis for ubiquitin after anti-Flag immunoprecipitation of HEK293T cells ectopically expressing Flag-GATA1 either alone or in combination with USP7-WT, and ubiquitin WT or mutant (K48R or K63R). 


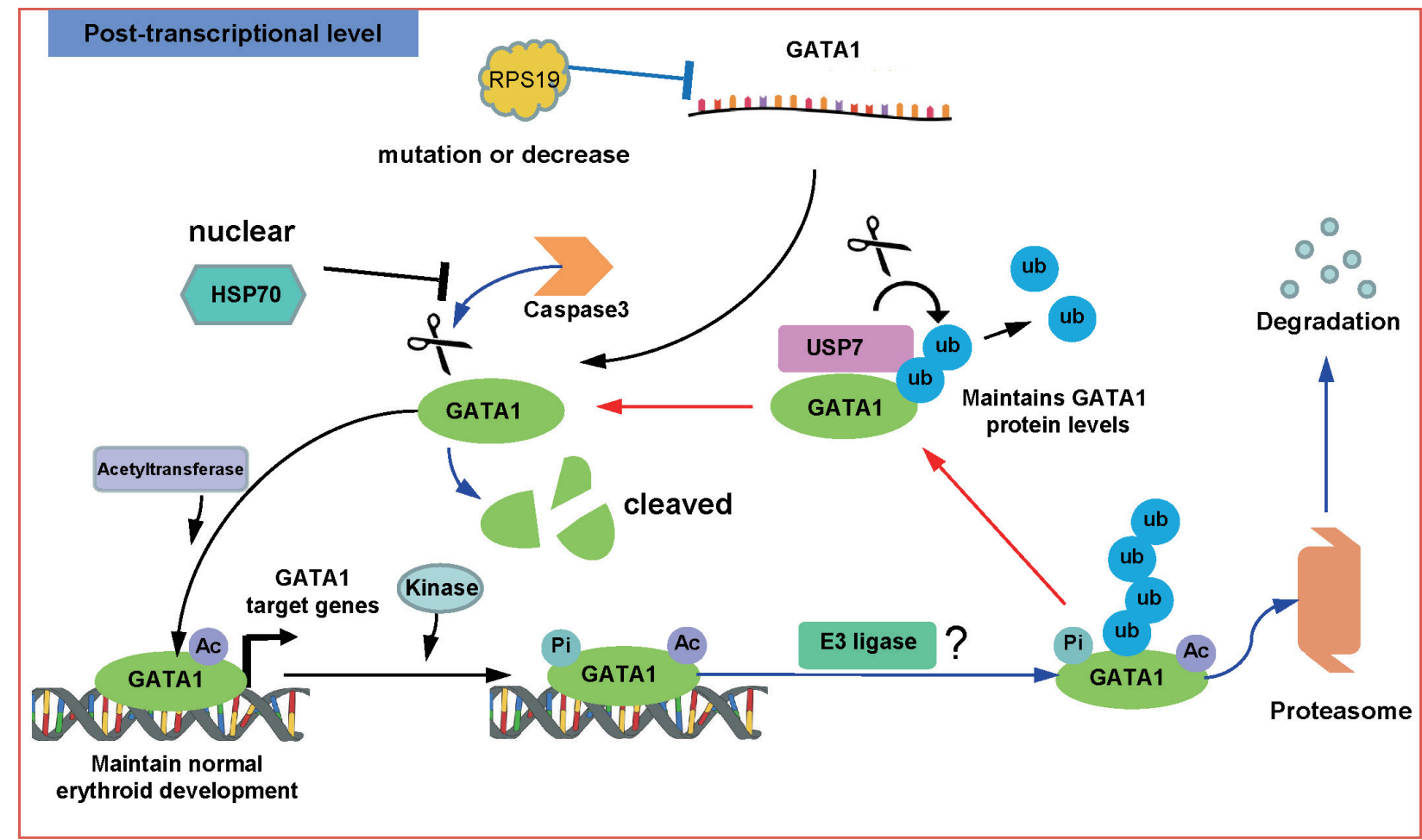

Figure 6. A schematic model of post-transcriptional regulation of GATA1. Post-transcriptional regulation of GATA1 includes the translational and post-translational levels. The translational level of GATA1 is mainly controlled by RPS19. Decrease or mutation of RPS19 results in reduced translation of GATA1. At the post-translational level, the nuclear HSP70 protects GATA1 from caspase 3 cleavage. In addition, acetylation and phosphorylation of GATA1 cooperate as the signal for ubiquitylation of GATA1 to degradation. USP7 interacts directly with GATA1 and maintains stability of GATA1 by removing the poly-ubiquitylation.

is known about the mechanisms by which the proteome is remodeled. Previous studies demonstrated that the balance between ubiquitination and deubiquitination plays important roles in homeostasis of cellular protein pools. ${ }^{45}$ In the present study, we documented, for the first time, the role of a deubiquitylase, USP7, in erythroid differentiation. We further documented that the mechanism is stabilization of the erythropoiesis master regulator GATA1.

USP7 is a member of a deubiquitinating enzyme family that contains more than 90 genes. ${ }^{46}$ USP7 expression is ubiquitous in different cell types: mice with knocked out USP7, which are homozygous for a null allele, show embryonic growth arrest and die between embryonic day 6.5 and 7.5. ${ }^{47}$ Furthermore, conditional knockout mice showed that USP7 is required for development of the central nervous system and functional regulatory $\mathrm{T}$ cells. ${ }^{48,49}$ We expect that deletion of USP7 in erythroid cells in vivo will led to altered erythropoiesis. We are in the process of generating such conditional knockout mice to define the function of USP7 in vivo. Besides USP7, many other deubiquitinating enzymes are also expressed in erythroid cells, although at lower levels than USP7. It will be interesting in future studies to identify the functional roles of other deubiquitylases during erythroid differentiation. Since each deubiquitinating enzyme has different substrate specificity ${ }^{47}$ it is likely that members of the deubiquitinating enzyme family may regulate different aspects of erythropoiesis via different mechanisms.

As the key transcriptional factor for erythropoiesis, GATA1 protein expression is tightly regulated at several levels. These include translational control by ribosome lev- els, ${ }^{26,41}$ stabilization by HSP70 from caspase 3 cleavage, ${ }^{27,38-40}$ and degradation by acetylation and phosphorylation-associated ubiquitination. ${ }^{24}$ Here we show that knockdown of USP7 by shRNA or inhibition of USP7 activity by USP7specific inhibitors led to dramatic decreases in GATA1 protein levels. Interestingly, USP7 knockdown or inhibition had no effects on the protein levels of RPS19 or HSP70, strongly suggesting that USP7 affects the stability of GATA1 in a direct manner. This notion is supported by our findings that USP7 binds GATA1 directly and stabilizes GATA1 by de-ubiquitination. Specifically, USP7 catalyzes the removal of K48-linked poly-ubiquitin which is a proteasome degradation signal for proteins. Based on our findings and that of others, we propose a schematic model for post-transcriptional regulation of GATA1 (Figure 6). GATA1 functions in the context of multi-protein complexes that include interacting proteins such as FOG1. ${ }^{50-53}$ Although USP7 knockdown or inhibition did not affect the level of FOG1 or NuRD complex (Online Supplementary Figure S8), we cannot exclude the possibility that these important GATA1 cofactors or modifications can modulate GATA1-USP7 interactions during erythroid development. Further study is therefore warranted to investigate whether USP7 binds other cofactors such as FOG1 and/or different modifications of GATA1.

Altered expression of GATA1 has been reported in myelodysplastic syndromes ${ }^{39,54}$ and $\beta$-thalassemia. ${ }^{40}$ However, the mechanisms of the altered GATA1 expression remains to be fully defined. It has been reported that USP7 is associated with several human diseases. ${ }^{55-57}$ Given the close relationship between USP7 and GATA1, demon- 
strated in our present study, it will be interesting in future studies to examine whether the altered expression of GATA1 may be associated with changes in USP7 expression in certain blood disorders.

In summary, we have uncovered a previously unrecognized role for a deubiquitylase, USP7, in human terminal erythroid differentiation and have identified USP7 as a deubiquitylase of GATA1. Our findings provide new and novel insights into mechanisms of regulating human erythropoiesis.

\section{Acknowledgments}

This work was supported by the National Key Research and Development Program of China (2018YFA0107800), the Natural Science Foundation of China (81770107,81672760, $81920108004,81270576,81800125,81470362$ and 81530005), National Institutes of Health grants (DK100810 and DK32094), the Strategic Priority Research Program of Central South University (zLXD2017004) and the postgraduate innovation project of Central South University (2016zzts165).

\section{References}

1. Hattangadi SM, Wong $\mathrm{P}$, Zhang $\mathrm{L}$, et al. From stem cell to red cell: regulation of erythropoiesis at multiple levels by multiple proteins, RNAs, and chromatin modifications. Blood. 2011;118(24):6258-6268.

2. An X, Schulz VP, Li J, et al. Global transcriptome analyses of human and murine terminal erythroid differentiation. Blood. 2014;123(22):3466-3477

3. Nandakumar SK, Ulirsch JC, Sankaran VG. Advances in understanding erythropoiesis: evolving perspectives. Br J Haematol. 2016;173(2):206-218

4. Dzierzak E, Philipsen S. Erythropoiesis: development and differentiation. Cold Spring Harb Perspect Med. 2013;3(4): a011601.

5. Amm I, Sommer T, Wolf DH. Protein quality control and elimination of protein waste: the role of the ubiquitin-proteasome system. Biochim Biophys Acta. 2014;1843 (1):182-196.

6. Ciechanover A, Hod Y, Hershko A. A heatstable polypeptide component of an ATPdependent proteolytic system from reticulocytes. Biochem Biophys Res Commun. 1978;81(4):1100-1105

7. Li B, Jia N, Kapur R, Chun KT. Cul4A targets $\mathrm{p} 27$ for degradation and regulates proliferation, cell cycle exit, and differentiation during erythropoiesis. Blood. 2006;107(11): 4291-4299.

8. Waning DL, Li B, Jia N, et al. Cul4A is required for hematopoietic cell viability and its deficiency leads to apoptosis. Blood. 2008;112(2):320-329

9. Nguyen AT, Prado MA, Schmidt PJ, et al. UBE2O remodels the proteome during terminal erythroid differentiation. Science. 2017;357(6350).

10. D'Andrea A, Pellman D. Deubiquitinating enzymes: a new class of biological regulators. Crit Rev Biochem Mol Biol. 1998;33(5):337-352.

11. Hanpude P, Bhattacharya S, Dey AK, Maiti TK. Deubiquitinating enzymes in cellular signaling and disease regulation. IUBMB Life. 2015;67(7):544-555.

12. Zlatanou A, Sabbioneda S, Miller ES, et al. USP7 is essential for maintaining Rad18 stability and DNA damage tolerance. Oncogene. 2016;35(8):965-976.

13. Van der Knaap JA, Kumar BR, Moshkin YM, et al. GMP synthetase stimulates histone $\mathrm{H} 2 \mathrm{~B}$ deubiquitylation by the epigenetic silencer USP7. Mol Cell. 2005;17(5):695707.

14. Alonso-de Vega I, Martín Y, Smits VA. USP7 controls Chk1 protein stability by direct deubiquitination. Cell Cycle. 2014;13 (24):3921-3926

15. Li M, Chen D, Shiloh A, et al. Deubiquitination of p53 by HAUSP is an important pathway for p53 stabilization. Nature. 2002;416(6881):648-653.

16. Daubeuf S, Singh D, Tan Y, et al. HSV ICPO recruits USP7 to modulate TLR-mediated innate response. Blood. 2009;113(14):3264 3275 .

17. Huang Z, Wu O, Guryanova OA, et al Deubiquitylase HAUSP stabilizes REST and promotes maintenance of neural progenitor cells. Nat Cell Biol. 2011;13(2):142-152.

18. Tang Y, Lv L, Li W, et al. Protein deubiquitinase USP7 is required for osteogenic differentiation of human adipose-derived stem cells. Stem Cell Res Ther 2017;8(1):186

19. Gao Y, Koppen A, Rakhshandehroo M, et al. Early adipogenesisis regulated through USP7-mediated deubiquitylation of the histone acetyltransferase TIP60. Nat Commun. 2013;4:2656.

20. Crispino JD. GATA1 in normal and malignant hematopoiesis. Semin Cell Dev Biol. 2005;16(1):137-147.

21. Fujiwara Y, Browne CP, Cunniff $K$, et al. Arrested development of embryonic red cell precursors in mouse embryos lacking transcription factor GATA-1. Proc Nat Acad Sci U S A. 1996;93(22):12355-12358.

22. Pevny L, Simon MC, Robertson E, et al. Erythroid differentiation in chimaeric mice blocked by a targeted mutation in the gene for transcription factor GATA-1. Nature. 1991; 349(6303): 257-260.

23. Whyatt D, Lindeboom F, Karis A, et al. An intrinsic but cell-nonautonomous defect in GATA-1-overexpressing mouse erythroid cells. Nature. 2000;406(6795):519-524.

24. Hernandez-Hernandez A, Ray P, Litos G, et al. Acetylation and MAPK phosphorylation cooperate to regulate the degradation of active GATA-1. EMBO J. 2006;25(14):3264 3274.

25. Hu J, Liu J, Xue F, et al. Isolation and functional characterization of human erythroblasts at distinct stages: implications for understanding of normal and disordered erythropoiesis in vivo. Blood. 2013;121(16) 3246-3253.

26. Ludwig LS, Gazda HT, Eng JC, et al. Altered translation of GATA1 in Diamond Blackfan anemia. Nat Med. 2014;20(7):748753.

27. Han X, Zhang J, Peng Y, et al. Unexpected role for p19INK4d in posttranscriptional regulation of GATA1 and modulation of human terminal erythropoiesis. Blood. 2017;129(2):226-237

28. Choo YS, Zhang Z. Detection of protein ubiquitination. J Vis Exp. 2009;(30)

29. Yan H, Wang Y, Qu X, et al. Distinct roles for TET family proteins in regulating human erythropoiesis. Blood. 2017;129 (14):2002-2012

30. Huang Y, Hale J, Wang Y, et al. SF3B1 deficiency impairs human erythropoiesis via activation of p53 pathway: implications for understanding of ineffective erythropoiesis in MDS. J Hematol Oncol. 2018;11(1):19.

31. Chauhan D, Tian Z, Nicholson B, et al. A small molecule inhibitor of ubiquitin-specific protease-7 induces apoptosis in multiple myeloma cells and overcomes bortezomib resistance. Cancer Cell. 2012;22(3): 345-358.

32. Altun M, Kramer H B, Willems L I, et al. Activity-based chemical proteomics accelerates inhibitor development for deubiquitylating enzymes. Chem Biol. 2011;18(11) 1401-1412.

33. Morotti A, Panuzzo C, Crivellaro S, et al BCR-ABL disrupts PTEN nuclear-cytoplasmic shuttling through phosphorylationdependent activation of HAUSP. Leukemia. 2014;28(6):1326-1333

34. Song MS, Salmena L, Carracedo A, et al. The deubiquitinylation and localization of PTEN are regulated by a HAUSP-PML network. Nature. 2008;455(7214):813-817.

35. Crossley M, Tsang A P, Bieker J J, et al. Regulation of the erythroid Kruppel-like factor (EKLF) gene promoter by the erythroid transcription factor GATA-1. J Biol Chem. 1994;269(22):15440-15444.

36. Kobayashi M, Yamamoto M. Regulation of GATA1 gene expression. J Biol Chem. 2007;142(1):1-10.

37. Kaneko H, Shimizu R, Yamamoto $M$ GATA factor switching during erythroid differentiation. Curr Opin Hematol. 2010; 17(3):163-168.

38. Ribeil JA, Zermati Y, Vandekerckhove J, et al. Hsp70 regulates erythropoiesis by preventing caspase-3-mediated cleavage of GATA-1. Nature. 2007;445(7123):102-105.

39. Frisan E, Vandekerckhove J, de Thonel A, et al. Defective nuclear localization of Hsp70 is associated with dyserythropoiesis and GATA-1 cleavage in myelodysplastic syndromes. Blood. 2012;119(6):1532-1542.

40. Arlet JB, Ribeil JA, Guillem F, et al. HSP70 sequestration by free $\alpha$-globin promotes ineffective erythropoiesis in $\beta$-thalas saemia. Nature. 2014;514(7521):242-246.

41. Khajuria RK, Munschauer M, Ulirsch JC, et al. Ribosome levels selectively regulate translation and lineage commitment in human hematopoiesis. Cell. 2018;173(1): 90-103.

42. de Thonel A, Vandekerckhove J, Lanneau D, et al. HSP27 controls GATA-1 protein 
level during erythroid cell differentiation. Blood. 2010;116(1):85-96.

43. Wu J, Zhou LQ, Yu W, et al. PML4 facilitates erythroid differentiation by enhancing the transcriptional activity of GATA-1. Blood. 2014;123(2):261-270.

44. Gautier EF, Ducamp S, Leduc M, et al. Comprehensive proteomic analysis of human erythropoiesis. Cell Rep. 2016;16(5):1470-1484

45. Clague MJ, Heride C, Urbé S. The demographics of the ubiquitin system. Trends Cell Biol. 2015;25(7):417-426.

46. Clague MJ, Barsukov I, Coulson JM, et al. Deubiquitylases from genes to organism. Physiol Rev. 2013;93(3):1289-1315.

47. Kon N, Kobayashi Y, Li M, etal. Inactivation of HAUSP in vivo modulates p53 function. Oncogene. 2010;29(9):1270-1279.

48. Kon N, Zhong J, Kobayashi Y, et al. Roles of HAUSP-mediated p53 regulation in central nervous system development. Cell Death
Differ. 2011;18(8):1366-1375

49. van Loosdregt J, Fleskens V, Fu J, et al. Stabilization of the transcription factor Foxp3 by the deubiquitinase USP7 increases Treg-cell-suppressive capacity. Immunity. 2013;39(2):259-271.

50. Crispino JD, Lodish MB, MacKay JP, Orkin $\mathrm{SH}$. Use of altered specificity mutants to probe a specific protein-protein interaction in differentiation: the GATA-1:FOG complex. Mol Cell. 1999;3(2):219-228.

51. Rodriguez P, Bonte E, Krijgsveld J, et al. GATA-1 forms distinct activating and repressive complexes in erythroid cells. EMBO J. 2005;24(13):2354-2366.

52. Tsang AP, Visvader JE, Turner CA, et al FOG, a multitype zinc finger protein, acts as a cofactor for transcription factor GATA1 in erythroid and megakaryocytic differentiation. Cell. 1997;90(1):109-119.

53. Miccio A, Wang Y, Hong W, et al. NuRD mediates activating and repressive func- tions of GATA-1 and FOG-1 during blood development. EMBO J. 2010;29(2):442-456. 54. Hopfer O, Nolte F, Mossner M, et al. Epigenetic dysregulation of GATA1 is involved in myelodysplastic syndromes dyserythropoiesis. Eur J Haematol. 2012;88 (2):144-153.

55. Hao YH, Fountain MD Jr, FonTacer K, et al. USP7 acts as a molecular rheostat to promote WASH-dependent endosomal protein recycling and is mutated in a human neurodevelopmental disorder. Mol Cell 2015;59(6):956-969

56. Nicholson B, Suresh Kumar KG. The multifaceted roles of USP7: new therapeutic opportunities. Cell Biochem Biophys. 2011;60(1-2):61-68.

57. YeasminKhusbu F, Chen FZ, Chen HC Targeting ubiquitin specific protease 7 in cancer: a deubiquitinase with great prospects. Cell Biochem Funct. 2018;36(5): 244-254. 